\title{
Планирование и оценка исследований биоэквивалентности препаратов эналаприла
}

\section{Д. П. Ромодановский1,*, А. Л. Хохлов², А. Е. Мирошников ${ }^{2}$}

${ }^{1}$ Федеральное государственное бюджетное учреждение «Научный центр экспертизы средств медицинского применения» Министерства здравоохранения Российской Федерации,

Петровский б-р, д. 8, стр. 2, Москва, 127051, Российская Федерация

${ }^{2}$ Федеральное государственное бюджетное образовательное учреждение высшего образования «Ярославский государственный медицинский университет» Министерства здравоохранения Российской Федерации, ул. Революционная, д. 5, Ярославль, 150000, Российская Федерация

Резюме. В настоящее время является актуальным проведение ретроспективного анализа исследований биоэквивалентности лекарственных средств определенного международного непатентованного наименования. За прошедшие годы в Российской Федерации накоплен достаточный пул данных, на основе которого можно провести анализ по выявлению многочисленных различий, сложностей и ошибок в исследованиях биоэквивалентности, что имеет особое значение для высоковариабельных лекарственных средств. Поскольку ингибиторы ангиотензин-превращающего фермента могут быть высоковариабельными, проведен ретроспективный анализ исследований биоэквивалентности воспроизведенных препаратов эналаприла отечественного и зарубежного производства. Представлены результаты данного анализа с целью определения коэффициентов внутрииндивидуальной вариабельности и унификации подходов к планированию и оценке исследований биоэквивалентности эналаприла. Показано, что препараты эналаприла не обладают высокой вариабельностью ни по исходному соединению, ни по активному метаболиту. Однако были выявлены многочисленные различия в проведенных исследованиях: например, набиралось разное количество здоровых добровольцев, определялась концентрация либо только исходного вещества, либо совместно с активным метаболитом, использовались различные аналитические методы определения аналитов, отличались длительность забора образцов крови и временные точки забора. Выявленные различия позволили унифицировать ключевые параметры исследований, гармонизировать их с актуальными требованиями к проведению исследований биоэквивалентности и сформулировать рекомендации в отношении планирования и оценки исследований эналаприла. При выборе дизайна исследований, помимо стандартных условий, можно рекомендовать следующее: количество здоровых добровольцев для проведения исследований биоэквивалентности эналаприла должно быть не менее 18; необходимо определять фармакокинетические параметры как эналаприла, так и его основного активного метаболита (эналаприлата); длительность определения аналитов в крови должна быть не менее 24 ч; достаточный период отмывки составляет 7 сут; точки забора крови с целью оценки максимальной концентрации должны быть более частыми в диапазоне времени около 1 ч для эналаприла и около 3 ч у эналаприлата.

Ключевые слова: исследование биоэквивалентности; высоковариабельные препараты; ингибиторы ангиотензин-превращающего фермента; эналаприл; эналаприлат

Для цитирования: Ромодановский ДП, Хохлов АЛ, Мирошников АЕ. Планирование и оценка исследований биоэквивалентности препаратов эналаприла. Ведомости Научного иентра экспертизы средств медицинского применения. 2018;8(4):231-237. https://doi.org/10.30895/1991-2919-2018-8-4-231-237

*Контактное лицо: Ромодановский Дмитрий Павлович; Romodanovsky@expmed.ru

\section{Planning and Evaluation of Bioequivalence Studies of Enalapril Products}

\section{P. Romodanovsky ${ }^{1, *}$, A. L. Khokhlov², A. E. Miroshnikov²}

${ }^{1}$ Scientific Centre for Expert Evaluation of Medicinal Products, 8/2 Petrovsky Blvd, Moscow 127051, Russian Federation ${ }^{2}$ Yaroslavl State Medical University, 5 Revolutsionnaya St., Yaroslavl 150000, Russian Federation

Abstract. Nowadays retrospective analysis of bioequivalence studies of medicinal products that have the same International Nonproprietary Name is becoming particularly relevant. Over the past years we have accumulated a sufficient pool of data in the Russian Federation which make it possible to identify many differences, difficulties and errors associated with bioequivalence studies (especially those of highly variable drugs). Given that inhibitors of angiotensin-converting enzyme (ACE) can be highly variable, we conducted a retrospective analysis of bioequivalence studies of domestically-produced and foreign-made generic enalapril products. This article presents the results of the analysis whose aim was to determine intraindividual coefficients of variation and harmonise approaches to planning and evaluation of enalapril bioequivalence studies. It was demonstrated that enalapril products do not show high variability both in terms of the original compound and the active metabolite. However, the analysis revealed many differences in the study designs: recruitment of a different number of healthy volunteers; determination of the concentration of either the original compound only, or in combination with the active metabolite; various analytical methods used for determination of analytes; different duration of blood sampling; and different sampling time points. The revealed differences made it possible to standardise the key research parameters, to harmonise them with the current requirements for conducting bioequivalence studies, and to formulate recommendations for planning and evaluation of enalapril studies. With respect to the study design, in addition to the standard conditions, the following can be recommended: at least 18 healthy volunteers should participate in enalapril bioequivalence studies; the pharmacokinetic parameters should be determined for both enalapril and its main active metabolite (enalaprilat); the duration of blood analyte determination should be at least 24 hours; the washout period should be 7 days; blood sampling for estimation of the maximum concentration should be more frequent around one hour after administration for enalapril and after three hours for enalaprilat.

Key words: bioequivalence study; highly variable drugs; inhibitors of angiotensin-converting enzyme; enalapril; enalaprilat

For citation: Romodanovsky DP, Khokhlov AL, Miroshnikov AE. Planning and evaluation of bioequivalence studies of enalapril products. Vedomosti Nauchnogo tsentra ekspertizy sredstv meditsinskogo primeneniya = The Bulletin of the Scientific Centre for Expert Evaluation of Medicinal Products. 2018;8(4):231-237. https://doi.org/10.30895/1991-2919-2018-8-4-231-237

*Corresponding author: Dmitry P. Romodanovsky; Romodanovsky@expmed.ru 
С регуляторной точки зрения среди множества лекарственных средств выделяют отдельную группу, которая характеризуется высокой вариабельностью таких фармакокинетических параметров, как максимальная концентрация в крови $\left(C_{\text {max }}\right)$ и площадь под кривой «концентрация - время» $(A U C)$. Такие лекарства называют лекарственными препаратами с высоковариабельными фармакокинетическими параметрами (или высоковариабельными), т.е. это препараты, у которых коэффициент внутрииндивидуальной вариабельности $\left(C V_{\text {intra }}\right)$ по крайней мере одного из двух основных параметров биоэквивалентности $\left(C_{\max }\right.$ и $\left.A U C\right)$ составляет $\geq 30 \%$. Другими словами, это препараты, скорость и степень всасывания которых демонстрируют высокую вариабельность у одного и того же субъекта при приеме лекарственного препарата в одинаковой дозировке [1-5].

Согласно анализу, проведенному экспертами Управления по контролю за качеством продуктов питания и лекарственных средств США (Food and Drug Administration, FDA), в общей структуре заявлений на регистрацию воспроизведенных лекарственных препаратов в $11 \%$ случаев по результатам исследований биоэквивалентности встречаются высоковариабельные препараты. Наиболее часто высоковариабельные препараты встречались в следующих фармакологических классах: ингибиторы ангиотензин-превращающего фермента (АПФ), блокаторы «медленных» кальциевых каналов, статины и бифосфонаты [3].

Одним из наиболее распространенных ингибиторов АПФ является эналаприл, который представляет собой пролекарство. В основном эналаприл применяют в кардиологии для лечения артериальной гипертензии, сердечной недостаточности, профилактики сердечной недостаточности и коронарной ишемии у пациентов с дисфункцией левого желудочка. Препараты эналаприла входят в список важнейших лекарственных средств Всемирной организации здравоохранения, а также в перечень жизненно необходимых и важнейших лекарственных препаратов ${ }^{1,2}$ Российской Федерации.

Учитывая социально-экономическую важность препаратов эналаприла, целесообразно разработать основополагающие научно-методические подходы к планированию дизайна и оценке результатов исследований терапевтической эквивалентности препаратов эналаприла, что позволит повысить качество проводимых исследований, а также облегчить задачу признания взаимозаменяемости препаратов эналаприла.

Одним из основных критериев для подтверждения терапевтической эквивалентности воспроизведенных препаратов является доказательство их биоэквивалентности [6, 7].

Цель работы - подготовка рекомендаций в отношении планирования и оценки исследований биоэквивалентности эналаприла, а именно: коли- чества субъектов для проведения исследования; необходимости определения исходного соединения или его основного активного метаболита; длительности периода отбора крови и выбора временных интервалов отбора крови; выбора аналитического метода определения аналитов; достаточного периода отмывки, а также оценки внутрииндивидуальной вариабельности эналаприла и его активного метаболита эналаприлата.

Для разработки таких научно-методических подходов необходимо провести анализ имеющихся требований к проведению фармакокинетических исследований in vivo препаратов эналаприла. Также необходим анализ результатов уже проведенных исследований эналаприла с оценкой $C V_{\text {intra }}$ фармакокинетических параметров. Обобщив полученные данные, можно определить, относится ли эналаприл к лекарственным средствам с высокой вариабельностью параметров фармакокинетики, и сформировать методические рекомендации в отношении исследований биоэквивалентности препаратов эналаприла.

\section{МАТЕРИАЛЫ И МЕТОДЫ}

1. В работе был использован информационно-аналитический метод для оценки имеющихся требований к проведению фармакокинетических исследований биоэквивалентности in vivo препаратов эналаприла по данным FDA, Европейского агентства по лекарственным средствам (European Medicines Agency, EMA), Всемирной организации здравоохранения (ВО3), Министерства здравоохранения Канады (Health Canada), Японского Национального института медицинских наук (NIHS), Администрации терапевтических товаров Министерства здравоохранения Австралии (TGA). Поиск выполнялся по запросам на официальных сайтах вышеуказанных регуляторных органов и в поисковой системе «Google» информационно-телекоммуникационной сети Интернет. Критерии поиска: «руководство», «биоэквивалентность», «эналаприл».

2. Применен ретроспективный анализ десяти отчетов исследований биоэквивалентности по материалам дел, поступивших в ФГБУ «НЦЭСМП» Минздрава России с целью регистрации, на основании результатов исследований биоэквивалентности эналаприла за период с 2006 по 2015 г. Проведен пересчет основных фармакокинетических параметров $C_{\max }$ и $A U C_{0-\mathrm{t}}$ из исходных данных о концентрациях эналаприла и/или эналаприлата исследуемых препаратов в сравнении с референтным препаратом. $A U C_{0-\mathrm{t}}$ рассчитывали методом трапеций в программе Microsoft Office Excel. Фармакокинетические параметры были $\log$-трансформированы и подвергнуты дисперсионному анализу (Analysis of variance, ANOVA) с помощью программы Statistica 10. В дисперсионный анализ были включены следующие факторы, вносящие вклад в наблюдаемую вариацию

WHO Model list of essential medicines. 20th edition. 2017.

Распоряжение Правительства Российской Федерации от 23 октября 2017 г. № 2323-р «Об утверждении перечня жизненно необходимых и важнейших лекарственных препаратов на 2018 год, а также перечней лекарственных препаратов для медицинского применения и минимального ассортимента лекарственных препаратов, необходимых для оказания медицинской помощи». 
данных: различия между препаратами, различия между субъектами (межиндивидуальные различия), последовательность приема препаратов, периоды исследования. На основании полученных значений были рассчитаны значения $C V_{\text {intra }}$

3. Использован информационно-аналитический метод оценки ключевых аспектов дизайна проведенных исследований биоэквивалентности.

\section{РЕЗУЛЬТАТЫ И ОБСУЖДЕНИЕ}

При планировании любого исследования биоэквивалентности необходимо провести анализ имеющихся данных о фармакологических свойствах действующего вещества и, в частности, сведений о фармакокинетике.

Фармакокинетические свойства эналаприла

Эналаприл является пролекарством, которое после перорального приема и абсорбции гидролизуется в печени, превращаясь в активное веществоэналаприлат. В кишечнике всасывается 60-70\% эналаприла, 60 \% которого трансформируется в эналаприлат. Общая биодоступность эналаприла в перерасчете на эналаприлат - около 40 \%. Абсорбция эналаприла не зависит от приема пищи. Максимальная концентрация эналаприла в крови достигается в течение 1 ч, а эналаприлата - через 3,5-4,5 ч после приема внутрь. Около 2/3 дозы эналаприла в виде неизмененного эналаприла и эналаприлата выводится почками, остальная часть дозы - через кишечник. Элиминация эналаприла из организма двухфазная: первая фаза с периодом полувыведения $\left(t_{1 / 2}\right) 2-6$ ч соответствует быстрой почечной экскреции циркулирующего в крови эналаприла и его активного метаболита; за ней следует вторая фаза (период полувыведения 36 ч), в ходе которой выводятся остатки препарата, распределенные в тканях и связанные с АПФ. Период полувыведения эналаприлата при курсовом применении составляет ${ }^{3,4} 11$ ч.

Таким образом, при проведении исследований биоэквивалентности препаратов эналаприла необходимо учитывать следующее:

- эналаприл является пролекарством (наибольшей активностью обладает метаболит эналаприлат), и, соответственно, может потребоваться определение как исходного вещества, так и метаболита;

- максимальная концентрация эналаприла наблюдается в течение 1 ч после приема, а концентрация эналаприлата - в течение 3,5-4,5 ч, т.е. точки забора образцов плазмы крови для исследования должны быть в указанном диапазоне значений $C_{\max }$;

- период полувыведения составляет 11 ч для эналаприлата (для эналаприла 2-6 ч), поэтому достаточный период отмывки предполагается в течение 7 сут; длительность забора образцов считается достаточной до 44 ч (4 периода $\left.t_{1 / 2}\right)$.

\section{Регуляторные требования}

Общепринято, что в исследованиях для определения биоэквивалентности после однократного приема исследуемой дозы основными параметрами для анализа являются $A U C_{0-\mathrm{t}}$ (площадь под кривой «концентрация - время» в интервале времени $0-\mathrm{t}), A U C_{0-\infty}$ (площадь под кривой «концентрация время» в интервале времени $0-\infty)$ и $C_{\max }$ (максимальная концентрация лекарственного вещества). Критерием для установления биоэквивалентности лекарственных препаратов для применения внутрь является отношение фармакокинетических параметров $C_{\max }$ и $A U C$ исходного вещества или активного метаболита исследуемого препарата к таковым препарата сравнения, которое должно лежать в заранее установленном 90 \% доверительном интервале. Однако по критерию необходимости изучения фармакокинетики активных метаболитов требования к проведению и оценке исследований биоэквивалентности несколько различаются в разных странах.

Регуляторные органы, такие как FDA, EMA, Health Canada обычно рекомендуют, чтобы определялась концентрация исходного вещества ${ }^{5,6,7}$. Основанием для этой рекомендации является то, что $A U C$ исходного вещества более чувствительна к изменениям, чем метаболиты, которые в большей степени отражают метаболизм, распределение и выведение. В случае с лекарственными препаратами, чьи метаболиты вносят значительный вклад в безопасность и/или эффективность применения препарата, некоторые регулирующие органы, такие как FDA и TPD, требуют для подтверждения биоэквивалентности определения, помимо исходного активного компонента, также и метаболитов в качестве дополнительной информации. Так как в ряде случаев активные метаболиты превосходят по своей фармакологической активности исходные вещества, т.е. эффективность и безопасность препарата в большей степени обусловлена концентрацией метаболита, чем концентрацией исходного вещества, то определение концентрации активных метаболитов может иметь большое клиническое значение [8].

Тем не менее позиция ЕМА в отношении рассмотрения активных метаболитов для оценки биоэквивалентности отличается от позиций FDA и TPD в том смысле, что требуется только анализ исходного вещества при проведении исследований биоэквивалентности. В текущем руководстве ЕМА по проведению исследований биоэквивалентности четко указывается, что $C_{\max }$ исходного соединения, как правило, более чувствительна в обнаружении различий между сравниваемыми препаратами в скорости поглощения, а не $C_{\max }$ метаболита. Также уточняется, что нет необходимости в определении активных метаболитов, если есть возможность определения концентрации исходного вещества.

Инструкция по медицинскому применению препарата Ренитек. https://grls.rosminzdrav.ru

Summary of product characteristics of Renitec/Innovace. http://www.mhra.gov.uk

Bioequivalence studies with pharmacokinetic endpoints for drugs submitted under an ANDA. United States Food and Drug Administration. 2013. Investigation of bioequivalence (CPMP/EWP/QWP/1401/98 Rev. 1). European Medicines Agency. 2010.

Comparative bioavailability standards: formulations used for systemic effects. Health Canada. 2012. 
Таблица 1. Общие сведения по исследованиям, включенным в сравнительный анализ

Table 1. General information on the studies covered by the comparative analysis

\begin{tabular}{|c|c|c|c|}
\hline Дизайн & Дозировка, мг & Референтный препарат & Количество добровольцев \\
\hline $2 \times 2$ & 10 & Ренитек $^{\circledR}$ & 18 \\
\hline $2 \times 2$ & 10 & Ренитек $^{\circledR}$ & 18 \\
\hline $2 \times 2$ & 10 & Ренитек $^{\circledR}$ & 18 \\
\hline $2 \times 2$ & 20 & Ренитек $^{\circledR}$ & 18 \\
\hline $2 \times 2$ & 10 & Ренитек $^{\circledR}$ & 18 \\
\hline $2 \times 2$ & 20 & Ренитек $^{\circledR}$ & 18 \\
\hline $2 \times 2$ & 10 & Ренитек $^{\circledR}$ & 28 \\
\hline $2 \times 2$ & 20 & Ренитек $^{\circledR}$ & 18 \\
\hline
\end{tabular}

Примечание. 2х2 - исследование с простым перекрестным дизайном с 2 периодами в 2 последовательностях.

По результатам анализа имеющихся требований к проведению фармакокинетических исследований биоэквивалентности in vivo препаратов эналаприла выявлено только одно полное совпадение в соответствии с критериями поиска - руководство по изучению биоэквивалентности препаратов эналаприла (эналаприла малеата) ${ }^{8}$, разработанное

Таблица 2. Сравнительные данные по графикам отбора образцов крови и периоду отмывки

Table 2. Comparative data on the blood sampling schedule and washout period

\begin{tabular}{|c|c|c|}
\hline $\begin{array}{c}\text { Длительность } \\
\text { отбора образцов, ч }\end{array}$ & Временные точки, ч & $\begin{array}{c}\text { Период } \\
\text { отмывки, сут }\end{array}$ \\
\hline 24 & $\begin{array}{c}0 ; 0,5 ; 1 ; 1,5 ; 2 ; 3 ; 4 ; 6 \\
8 ; 12 ; 24\end{array}$ & 7 \\
\hline 24 & $\begin{array}{c}0,5 ; 1 ; 2 ; 3 ; 4 ; 6 ; 8 ; 12 ; \\
15 ; 24\end{array}$ & 7 \\
\hline 24 & $\begin{array}{c}0 ; 1 ; 2 ; 3 ; 4 ; 6 ; 10 ; 12 ; \\
24\end{array}$ & 7 \\
\hline 48 & $0 ; 2 ; 3 ; 4 ; 10 ; 24 ; 32 ; 48$ & 4 \\
\hline 30 & $\begin{array}{l}0 ; 0,5 ; 1 ; 1,5 ; 2 ; 3 ; 3,5 ; \\
\quad 4 ; 5 ; 8 ; 12 ; 24 ; 30\end{array}$ & 14 \\
\hline 24 & $\begin{array}{c}0 ; 0,5 ; 1 ; 2 ; 4 ; 6 ; 10 \\
12 ; 24\end{array}$ & Нет данных \\
\hline 30 & $\begin{array}{c}0 ; 1 ; 2 ; 2,5 ; 3 ; 3,5 ; 4 ; 5 \\
7 ; 10 ; 24 ; 30\end{array}$ & 7 \\
\hline 48 & $\begin{array}{l}0 ; 0,25 ; 0,5 ; 0,75 ; 1 ; \\
1,25 ; 1,5 ; 1,75 ; 2 ; 2,5 \\
3 ; 3,33 ; 3,67 ; 4 ; 4,5 ; 5 \\
\quad 6 ; 8 ; 12 ; 16 ; 24 ; 48\end{array}$ & 21 \\
\hline 24 & $\begin{array}{c}0 ; 0,17 ; 0,33 ; 0,66 ; 1 ; \\
1,5 ; 2 ; 3 ; 4 ; 6 ; 9 ; 12 ; 24\end{array}$ & 7 \\
\hline 48 & $\begin{array}{c}0 ; 0,5 ; 2 ; 3 ; 4 ; 5 ; 8 ; 12 \\
24 ; 48\end{array}$ & 7 \\
\hline
\end{tabular}

FDA. Во всех остальных случаях результатом поиска были общие руководства к исследованиям биоэквивалентности или статьи, посвященные проведенным исследованиям биоэквивалентности эналаприла. Согласно требованиям FDA, определяют и исходное вещество, и его метаболит, однако биоэквивалентность должна быть подтверждена для исходного соединения. Исследование рекомендуется проводить в условиях натощак и после приема пищи для дозировки 20 мг.

Ретроспективный анализ результатов исследований биоэквивалентности эналаприла с 2006 до 2015 г.

Проанализировано 10 наборов данных исследований биоэквивалентности препаратов эналаприла (табл. 1).

В исследованиях биоэквивалентности изучались дозы эналаприла 10 и 20 мг.

Количество здоровых добровольцев в различных исследованиях варьировало от 18 до 28, однако в большинстве случаев (в 8 из 10 исследований) количество здоровых добровольцев было равно 18 (табл. 1).

Длительность периода отбора образцов крови для фармакокинетического анализа варьировала от 24 ч $(\mathrm{n}=5)$ до 48 ч $(\mathrm{n}=3)$. Длительность периода отмывки составляла от 7 сут $(\mathrm{n}=6)$ до 21 сут $(\mathrm{n}=1)$ (табл. 2).

Среднее время достижения максимальной концентрации после приема воспроизведенных препаратов и референтного препарата составило для эналаприла $-0,89 \pm 0,22$ и 0,92 $\pm 0,2$ ч, для эналаприлата $-3,26 \pm 0,25$ и 3,39 $\pm 0,31$ ч соответственно.

В большинстве случаев для определения эналаприла и эналаприлата в плазме крови использовались методы высокоэффективной жидкостной хроматографии (ВЭЖХ) с масс-спектрометрической

8 Draft guidance on enalapril maleate. United States Food and Drug Administration. 2008. 
Таблица 3. Сравнительные данные по аналитическому методу и нижнему пределу количественного определения эналаприла и энаприлата

Table 3. Comparative data on the analytical methods and lower limit of quantification for enalapril and enalaprilat

\begin{tabular}{|c|c|c|}
\hline $\begin{array}{c}\text { Аналитический } \\
\text { метод }\end{array}$ & Аналит & $\begin{array}{c}\text { НПКО, } \\
\text { нг/м. }\end{array}$ \\
\hline $\begin{array}{c}\text { Радиофермент- } \\
\text { ный анализ }\end{array}$ & Эналаприлат & 4 \\
\hline ВЭЖХ-УФ & Эналаприлат & 10 \\
\hline ИФА & Эналаприлат & 0,1 \\
\hline ВЭЖХ-МС & Эналаприлат & 2 \\
\hline ВЭЖХ-МС & $\begin{array}{c}\text { Эналаприл } \\
\text { и эналаприлат }\end{array}$ & $0,5^{*}$ \\
\hline ИФА & Эналаприлат & 0,03 \\
\hline ИФА & Эналаприлат & Нет данных \\
\hline ВЭЖХ-МС & $\begin{array}{c}\text { Эналаприл } \\
\text { и эналаприлат }\end{array}$ & $5^{*}$ \\
\hline ВЭЖХ-МС & $\begin{array}{c}\text { Эналаприл } \\
\text { и эналаприлат }\end{array}$ & $1^{*}$ \\
\hline ВЭЖХ-МС & $\begin{array}{c}\text { Эналаприл } \\
\text { и эналаприлат }\end{array}$ & $5^{*}$ \\
\hline
\end{tabular}

Примечание. ВЭЖХ-УФ - высокоэффективная жидкостная хроматография с ультрафиолетовой детекцией; ВЭЖХ-МС высокоэффективная жидкостная хроматография с массспектрометрической детекцией; ИФА - иммуноферментный анализ; НПКО - нижний предел количественного определения; * - значения НПКО применимы для эналаприла и эналаприлата.

детекцией $(\mathrm{n}=5)$ и иммуноферментный анализ $(\mathrm{n}=3)$. Нижний предел количественного определения варьировал от 0,03 до 10 нг/мл (табл. 3).

Во всех исследованиях внутрииндивидуальная вариабельность фармакокинетических показателей $\left(C_{\max }, A U C\right)$ не превышала $30 \%$, в ряде исследований наибольшая вариабельность была показана для эналаприлата - 25-26 \% (табл. 4 и 5).

Таким образом, значения внутрииндивидуального коэффициента вариабельности для

Таблица 4. Сравнительные данные по коэффициентам внутрииндивидуальной вариабельности эналаприла

Table 4. Comparative data on intraindividual coefficients of variation for enalapril

\begin{tabular}{|c|c|c|}
\hline Аналит & $\boldsymbol{C} \boldsymbol{V}_{\text {intra }} \boldsymbol{A U C}, \%$ & $\boldsymbol{C} \boldsymbol{V}_{\text {intra }} \boldsymbol{C}_{\max }, \%$ \\
\hline Эналаприл & 14,21 & 17 \\
\hline Эналаприл & 17,63 & 21,74 \\
\hline Эналаприл & 10,51 & 15,23 \\
\hline Эналаприл & 16,16 & 22,51 \\
\hline Среднее значение & 14,63 & 19,12 \\
\hline
\end{tabular}

Примечание. $C V_{\text {intra }}$ - коэффициент внутрииндивидуальной вариабельности; $A U C$ - площадь под кривой «концентрация время»; $C_{\max }-$ максимальная концентрация в плазме крови.
Таблица 5. Коэффициенты внутрииндивидуальной вариабельности эналаприлата

Table 5. Intraindividual coefficients of variation for enalaprilat

\begin{tabular}{|c|c|c|}
\hline Аналит & $\boldsymbol{C} \boldsymbol{V}_{\text {intra }} A \boldsymbol{A}, \%$ & $\boldsymbol{C} \boldsymbol{V}_{\text {intra }} \boldsymbol{C}_{\max } \%$ \\
\hline Эналаприлат & 7,75 & 17,74 \\
\hline Эналаприлат & 4,47 & 5,48 \\
\hline Эналаприлат & 16,54 & 17,74 \\
\hline Эналаприлат & 15,25 & 12,69 \\
\hline Эналаприлат & 8,35 & 12,92 \\
\hline Эналаприлат & 12,16 & 10,43 \\
\hline Эналаприлат & 8,56 & 15,28 \\
\hline Эналаприлат & 25,82 & 24,43 \\
\hline Эналаприлат & 3,21 & 12,53 \\
\hline Эналаприлат & 20,45 & 15,91 \\
\hline Среднее значение & 12,256 & 14,515 \\
\hline
\end{tabular}

Примечание. $C V_{\text {intra }}$ - коэффициент внутрииндивидуальной вариабельности; $A U C$ - площадь под кривой «концентрация - время»; $C_{\max }-$ максимальная концентрация в плазме крови.

параметров $C_{\max }$ и $A U C$ эналаприла и эналаприлата свидетельствуют о невысокой вариабельности препарата (средние значения около 19 и $14 \%$ по наиболее вариабельному параметру $C_{\max }$ соответственно). То есть среди ингибиторов АПФ эналаприл не является высоковариабельным, однако, учитывая ранее проведенный анализ FDA [3], целесообразно продолжить анализ данной группы препаратов для выявления высоковариабельных лекарственных средств.

Согласно данным портала Государственного реестра лекарственных средств и анализа проведенных исследований биоэквивалентности, в качестве референтного препарата в исследованиях биоэквивалентности следует использовать препарат Ренитек ${ }^{\circledR}$.

Учитывая зарубежные и отечественные требования к исследованиям биоэквивалентности, следует определять концентрацию исходного соединения - эналаприла. В дополнение к определению исходного вещества также необходимо определять концентрацию метаболита - эналаприлата. Биоэквивалентность должна быть подтверждена для исходного соединения.

Определять в крови эналаприл и эналаприлат рекомендуется методом ВЭЖХ с массспектрометрической детекцией, достаточно чувствительным и точным аналитическим методом, наиболее часто используемым для определения эналаприла и его метаболита согласно проанализированным исследованиям. Аналитическая методика должна быть надлежащим образом валидирована в соответствии с Правилами проведения исследований биоэквивалентности лекарствен- 
ных препаратов ${ }^{9}$ в рамках Евразийского экономического союза.

Рекомендуется проведение исследования для дозировки 20 мг, являющейся, как правило, максимальной и наиболее чувствительной к выявлению различий.

Учитывая среднее значение $C V_{\text {intra }}$ эналаприла около $19 \%$ (по результатам 4 исследований), для достижения $80 \%$ мощности исследования достаточно включения 18 субъектов.

Длительность определения концентрации эналаприла в плазме крови, учитывая данные по фармакокинетике, должна быть не менее 24 часов, период отмывки не менее 7 дней.

При выборе временных интервалов отбора крови следует учитывать более частый отбор образцов крови в диапазоне времени достижения $C_{\max }$ (около 1 ч для эналаприла и около 3 ч у эналаприлата). Можно порекомендовать следующий график отбора образцов крови: $0 ; 0,25 ; 0,5 ; 0,75 ; 1 ; 1,25 ; 1,5 ; 1,75 ; 2$; 2,$5 ; 3 ; 3,25 ; 3,5 ; 3,75 ; 4 ; 4,5 ; 5 ; 6 ; 8 ; 12 ; 16 ; 24$ ч.

Оценивать результаты необходимо в соответствии с общими требованиями для исследований биоэквивалентности $^{10}-90 \%$ доверительные интервалы для отношения средних значений $C_{\max }$ и $A U C$ эналаприла должны находиться в диапазоне 80-125\%.

\section{ЗАКЛЮЧЕНИЕ}

Препараты эналаприла не являются высоковариабельными.

Проведенный анализ позволяет сформулировать следующие положения и рекомендации в отношении планирования и оценки исследований биоэквивалентности препаратов эналаприла:

1. Референтный препарат - Ренитек ${ }^{\circledR}$.

2. Дозировка - 20 мг.

3. Количество добровольцев -18 .

4. Длительность и временные интервалы отбора крови - $0 ; 0,25 ; 0,5 ; 0,75 ; 1 ; 1,25 ; 1,5 ; 1,75 ; 2 ; 2,5 ; 3$; 3,$25 ; 3,5 ; 3,75 ; 4 ; 4,5 ; 5 ; 6 ; 8 ; 12 ; 16 ; 24$ ч.

5. Период отмывки -7 сут.

6. Аналит - эналаприл, эналаприлат.

7. Метод определения аналитов - ВЭЖХ-МС или ВЭЖХ-МС/МС.

Благодарности. Работа выполнена в рамках государственного задания ФГБУ «НЦЭСМП» Минздрава России № 056-00023-18-02 на проведение прикладных научных исследований (номер государственного учета НИР AAAA-A18-118021590049-0).

Acknowledgements. The study reported in this publication was carried out as part of a publicly funded research project No. 056-00023-18-02 and was supported by the Scientific Centre for Expert Evaluation of Medicinal Products (R\&D public accounting No. AAAA-A18-118021590049-0).

Конфликт интересов. Авторы заявляют об отсутствии конфликта интересов, требующего раскрытия в данной статье. Conflict of interest. Authors declare no conflict of interest requiring disclosure in this article.

\section{ЛИТЕPATYPA / REFERENCES}

1. Ромодановский ДП, Драницына МА, Горячев ДВ, Ниязов РР, Гавришина ЕВ. Планирование дизайна и оценка результатов исследований биоэквивалентности высоковариабельных препаратов на примере розувастатина. Экспериментальная и клиническая фармакология. 2015;78(6):19-25. [Romodanovsky DP, Dranitsyna MA, Goryachev DV, Niyazov RR, Gavrishina EV. Design planning and evaluation of the results of bioequivalence studies of highly variable drugs using rosuvastatin as an example. Eksperimental'naya $i$ klinicheskaya farmakologiya $=$ Experimental and Clinical Pharmacology. 2015;78(6):19-25 (In Russ.)] https://doi. org/10.30906/0869-2092-2015-78-6-19-25

2. Ромодановский ДП, Еременкова ТВ, Драницына МА, Горячев ДВ, Ниязов РР, Гавришина ЕВ, Меркулов ВА. Высоковариабельные лекарственные препараты - особенности исследования биоэквивалентности. Ведомости Научного чентра экспертизы средств медицинского применения. 2015;(4):5-10. [Romodanovsky DP, Eremenkova TV, Dranitsyna MA, Goryachev DV, Niyazov RR, Gavrishina EV, Merkulov VA. Highly variable medicines - specific aspects of bioequivalence studies. Vedomosti Nauchnogo tsentra ekspertizy sredstv meditsinskogo primeneniya $=$ The Bulletin of the Scientific Centre for Expert Evaluation of Medicinal Products. 2015;(4):5-10 (In Russ.)]

3. Davit BM, Conner DP, Fabian-Fritsch B, Haidar SH, Jiang X, Patel DT, et al. Highly variable drugs: observations from bioequivalence data submitted to the FDA for new generic drug applications. AAPS J. 2008;10(1):148-56. https://doi.org/10.1208/s12248-008-9015-x

4. Davit BM, Chen ML, Conner DP, Haidar SH, Kim S, Lee CH, et al. Implementation of a reference-scaled average bioequivalence approach for highly variable generic drug products by the US Food and Drug Administration. AAPS J. 2012;14(4):915-24. https://doi. org/10.1208/s12248-012-9406-X

5. Tothfalusi L, Endrenyi L, Arieta AG. Evaluation of bioequivalence for highly variable drugs with scaled average bioequivalence. Clin Pharmacokinet. 2009;48:725-43. https://doi.org/10.2165/11318040000000000-00000.

6. Жердев ВП, Колыванов ГБ, Литвин АА, Сариев АК. Гармонизация проведения исследований биоэквивалентности лекарственных препаратов: вопросы и их возможное решение. Экспериментальная и клиническая фармакология. 2003;66(2):60-4. [Zherdev VP, Kolyvanov GB, Litvin AA, Sariev AK. Harmonization of testing drugs for bioequivalence: problems and solutions. Eksperimentalnaya $i$ klinicheskaya farmakologiya = Experimental and Clinical Pharmacology. 2003;66(2):60-4 (In Russ.)] https://doi. org/10.30906/0869-2092-2003-66-2-60-64

7. Смирнов АС, Шнайдер А, Фролов МЮ, Петров ВИ. Современные критерии исследований биоэквивалентности лекарственных средств: гармонизация национальных стандартов. Химико-фармацевтический журнал. 2014;48(5):3-10. [Smirnov AS, Schnei$\operatorname{der}$ A, Frolov MYu, Petrov VI. Modern criteria of bioequivalence of pharmaceuticals: harmonization of national standards. Khimikofarmatsevticheskiy zhurnal $=$ Pharmaceutical Chemistry Journal. 2014;48(5):3-10 (In Russ.)]

8. Ромодановский ДП, Горячев ДВ. Актуальные аспекты планирования дизайна исследований биоэквивалентности препаратов аторвастатина. Ведомости Научного чентра экспертизы средств медицинского применения. 2013;(1):22-7. [Romodanovsky DP, Goryachev DV. Current aspects of planning bioequivalence studies design for atorvastatin preparations. Vedomosti Nauchnogo tsentra ekspertizy sredstv meditsinskogo primeneniya $=$ The Bulletin of the Scientific Centre for Expert Evaluation of Medicinal Products. 2013;(1):22-7 (In Russ.)]

Правила проведения исследований биоэквивалентности лекарственных средств Евразийского экономического союза, утвержденные Решением Совета Евразийской экономической комиссии от 3 ноября 2016 г. № 85.

10 Руководство по экспертизе лекарственных средств. Том I. М.: Гриф и К; 2013. 


\section{ОБ АВТОРАХ}

Ромодановский Дмитрий Павлович, канд. мед. наук, главный эксперт управления № 2 по эффективности и безопасности лекарственных средств Центра экспертизы и контроля готовых лекарственных средств ФГБУ «НЦЭСМП» Минздрава России, ORCID: http://orcid. org/0000-0002-2980-4518

Хохлов Александр Леонидович, д-р мед. наук, проф., член-корр. РАН, заведующий кафедрой клинической фармакологии с курсом Института последипломного образования ЯГМУ, ORCID: http://orcid. org/0000-0002-0032-0341

Мирошников Алексей Евгеньевич, канд. мед. наук, доцент кафедры клинической фармакологии с курсом Института последипломного образования ЯГМУ, ORCID: http://orcid.org/0000-0001-66573950

Статья поступила 12.03.2018

После доработки 14.06.2018

Принята к печати 19.11.2018

\section{AUTHORS}

Dmitry P. Romodanovsky, Cand. Sci. (Med.), Chief Expert of Division No. 2 on Medicinal Products' Efficacy and Safety of the Centre for Evaluation and Control of Medicinal Products of the Scientific Centre for Expert Evaluation of Medicinal Products, ORCID: http://orcid.org/0000-00022980-4518

Alexander L. Khokhlov, Dr. Sci. (Med.), Professor, Corresponding Member of RAS, Head of the Department of Clinical Pharmacology with the Course of Institute of Postgraduate Education of Yaroslavl State Medical University, ORCID: http://orcid.org/0000-0002-0032-0341

Aleksey E. Miroshnikov, Cand. Sci. (Med.), Assistant Professor of the Department of Clinical Pharmacology with the Course of Institute of Postgraduate Education of Yaroslavl State Medical University, ORCID: http://orcid.org/0000-0001-6657-3950

Article was received 12 March 2018

Revised 14 June 2018

Accepted for publication 19 November 2018 